\title{
A variability analysis of low-latitude unidentified gamma-ray sources ${ }^{\star}$
}

\author{
D. F. Torres ${ }^{1}$, G. E. Romero ${ }^{1, \star \star}$, J. A. Combi ${ }^{1, \star \star}$, P. Benaglia $^{1, \star \star}$, H. Andernach ${ }^{2}$, and B. Punsly ${ }^{3}$ \\ 1 Instituto Argentino de Radioastronomía, C.C.5, (1894) Villa Elisa, Buenos Aires, Argentina \\ 2 Depto. de Astronomía, Univ. Guanajuato, Apartado Postal 144, Guanajuato, CP 36000, GTO, Mexico \\ 34014 Emerald Street No. 116, Torrance, CA 90503, USA
}

Received 4 August 2000 / Accepted 26 January 2001

\begin{abstract}
We present a study of 40 low-latitude unidentified 3EG gamma-ray sources which were found to be not positionally coincident with any known class of potential gamma-ray emitters in the Galaxy (Romero et al. 1999). We have performed a variability analysis which reveals that many of these 40 sources are variable. These sources have, in addition, a steep mean value of the gamma-ray spectral index, $\langle\Gamma\rangle=2.41 \pm 0.2$, which, combined with the high level of variability, seems to rule out a pulsar origin. The positional coincidences with uncatalogued candidates to supernova remnants were also studied. Only 7 sources in the sample are spatially coincident with these candidates, a result that is shown to be consistent with the expected level of pure chance association. A complementary search for weak radio counterparts was also conducted and the results are presented as an extensive table containing all significant point-like radio sources within the 40 EGRET fields. We argue that in order to produce the high variability, steep gamma-ray spectra, and absence of strong radio counterparts observed in some of the gamma-ray sources of our sample, a new class of objects should be postulated, and we analyze a viable candidate.
\end{abstract}

Key words. gamma-rays: observations - gamma-rays: theory - ISM: supernova remnants - black holes physics

\section{Introduction}

The main results of the very successful Energetic Gamma Ray Telescope (EGRET) of the Compton Gamma Ray Observatory (CGRO) are contained in the Third EGRET (3EG) catalog (Hartman et al. 1999), which includes observations carried out between 22 April 1991 and 3 October 1995, and lists 271 point sources. Of these sources, 170 have no conclusive counterparts at lower frequencies. In the latitude range $|b| \leq 10^{\circ}$, there are 81 unidentified sources, a number which doubles that reported in the previous 2EG catalog and its supplement (Thompson et al. 1995, 1996), and clearly confirms the existence of a population of gamma-ray emitters in the Galaxy.

The nature of this population of low-latitude gammaray sources is not clear. Several types of objects have been proposed as possible counterparts in the past: supernova

\footnotetext{
Send offprint requests to: D. F. Torres,

e-mail: dtorres@venus.fisica.unlp.edu.ar

* Tables 5-8 are only available in electronic form at the CDS via anonymous ftp to cdsarc.u-strasbg.fr (130.79.128.5) or via

http://cdsweb.u-strasbg.fr/cgi-bin/qcat?J/A+A/370/468

$\star \star$ Member of CONICET.
}

remnants (e.g. Sturner \& Dermer 1995; Esposito et al. 1996; Combi et al. 1998a), massive stars with strong winds (e.g. Montmerle 1979; Romero et al. 1999; Benaglia et al. 2000), young pulsars (e.g. Yadigaroglu \& Romani 1997), and compact clouds in star-forming regions (e.g. Cassé \& Paul 1980). Gehrels et al. (2000) have recently shown that there is a population of sources associated with the Gould belt, as it was originally suggested by Grenier (1995). This suggests that young stellar objects play an important role in the generation of the observed emission.

In a recent paper (Romero et al. 1999, hereafter Paper I), some of us presented a study of the level of positional correlation between gamma-ray sources in the 3EG cata$\log$ and a variety of galactic objects. A statistically very significant correlation was found in the case of SNRs and OB star associations. In that work it was also found that a group of 42 low-latitude 3EG sources do not present a likely counterpart. Two of these sources were finally discovered to be artifacts related to the high intensity of the Vela pulsar (Hartman 2000). Very recently, Zhang et al. (2000) proposed that most of the sources spatially coincident with SNRs and OB associations could be pulsars. They have presented a variability study of the 38 3EG sources that are coincident with objects of these classes, arguing that most of them are not variable. 
In this work we focus our attention on the group of 40 gamma-ray sources which are not coincident with any known galactic object thought to be capable of producing gamma-ray emission. Classical galactic counterparts are expected to be non-variable on timescales of a few years and, consequently, we shall discuss the time behavior of the sources in our sample. The structure of the paper is as follows. In Sect. 2 we define the sample. A variability analysis is presented in Sect. 3. Section 4 deals with the possible positional correlation between sources in our sample and SNR candidates not included in Green's (1998) catalog. In Sect. 5 we explore possible weak radio counterparts of the gamma-ray sources and present an extensive table with all significant point-like radio sources within the 40 EGRET positional error boxes that, we expect, will be very helpful for future research in this field. In Sect. 6 we discuss the case for isolated Kerr-Newman black holes as possible generators of some of the gamma-ray sources, and finally, in Sect. 7, we draw our conclusions. Three appendices present detailed tables with data used in our research.

\section{Sample}

From the original set of 81 (75 if we exclude the six sources thought to be artifacts produced by the Vela pulsar) unidentified sources at $|b| \leq 10^{\circ}$, an apparently large fraction of $\sim 50 \%$ (40 sources) remain without any known likely galactic counterpart. We list these sources in Table 1, where we give their 3EG name, their galactic coordinates, the error in their position - assumed to be represented by the $95 \%$ confidence contours given by the 3EG catalog -, and their gamma spectral index $\Gamma$ (such that the photon distribution is given by $N(E) \propto E^{-\Gamma}$ ). We also present four further columns related to the variability analysis that will be explained below. A †-symbol on the source name indicates a source which was suggested as possible AGN by Hartman et al. (1999). It is worth noticing that the mean value of the spectral index is quite steep for these sources: $2.41 \pm 0.2$, steeper that the steepest pulsar spectrum known (Thompson et al. 1994). The distribution of the spectral index is shown in Fig. 1. Only three sources out of 40 have $\Gamma<2$.

\section{Gamma-ray variability}

We shall now assess the possible long term variability (on time-scale of years) of the sources in our sample. We will adopt the following criterion. We define a mean weighted value for the EGRET flux as:

$\langle F\rangle=\left[\sum_{i=1}^{N_{\mathrm{vp}}} \frac{F(i)}{\epsilon(i)^{2}}\right] \times\left[\sum_{i=1}^{N_{\mathrm{vp}}} \frac{1}{\epsilon(i)^{2}}\right]^{-1}$.

Here, $N_{\mathrm{vp}}$ is the number of viewing periods for each gamma-ray source. We take into account only single viewing periods (i.e. periods like $305+$, virgo, or the combined P12, P123, and P1234 were not taken into

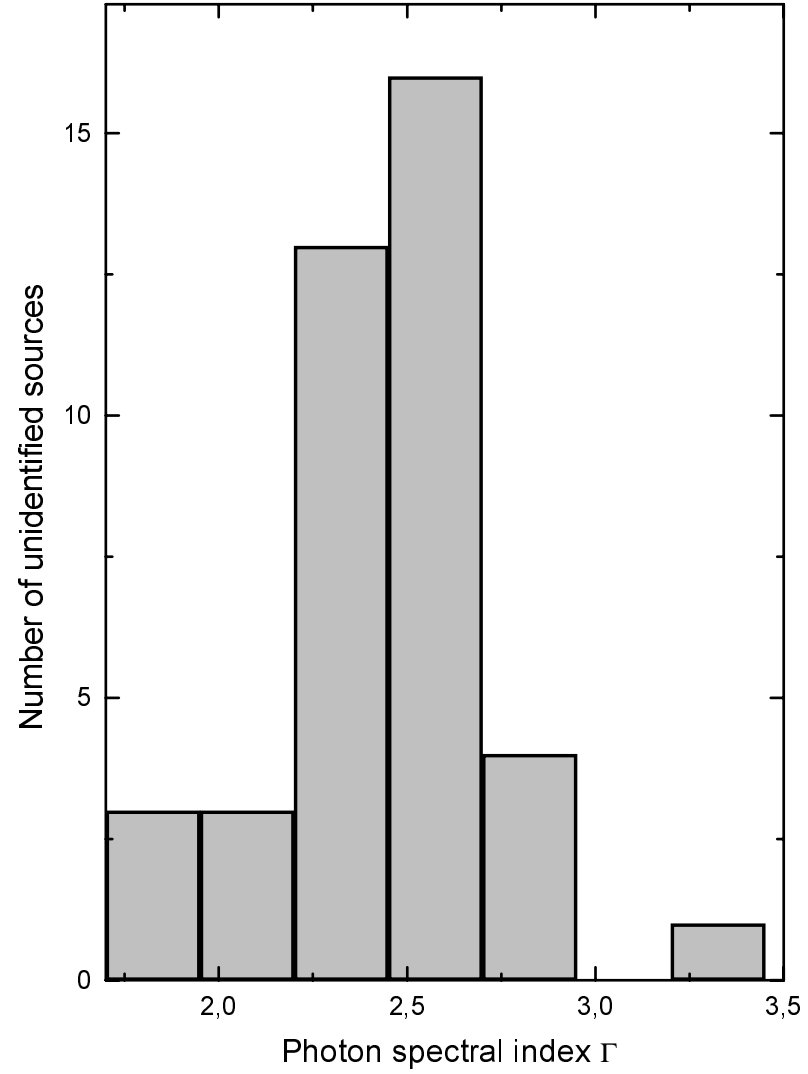

Fig. 1. Distribution of the spectral indices of the 40 unidentified sources

account). $F(i)$ is the observed flux in the $i$ th-period, whereas $\epsilon(i)$ is the corresponding error in the observed flux. We have taken these data directly from the 3EG catalog. For those observations in which the significance $(\sqrt{T S}$ in the EGRET catalog) is greater than $3 \sigma$, we took the error as $\epsilon(i)=F(i) / \sqrt{T S}$. However, many of the observations are in fact upper bounds on the flux, with significance below $3 \sigma$. For these ones, we assume both $F(i)$ and $\epsilon(i)$ as half the value of the upper bound. We then define the fluctuation index $\mu$ as:

$\mu=100 \times \sigma_{\mathrm{sd}} \times\langle F\rangle^{-1}$.

In this expression, $\sigma_{\mathrm{sd}}$ is the standard deviation of the flux measurements, taking into account the previous considerations.

Results for the 40 sources are given in Table 1, where we show four columns corresponding to $\langle F\rangle, \sigma_{\mathrm{sd}}, N_{\mathrm{vp}}$ and $\mu$.

In order to remove as far as possible spurious variability introduced by the observing system, we computed the fluctuation index $\mu$ for the confirmed gamma-ray pulsars in the 3EG catalog (see Cusumano et al. 2000 for the latest identification). We adopt the physical criterion that pulsars are - i.e. by definition - non-variable gammaray sources. Then, any non-null $\mu$-value for pulsars is attributed to experimental uncertainty. We then define an averaged statistical index of variability, $I$, as

$I=\frac{\mu_{\text {source }}}{\left\langle\mu>_{\text {pulsars }}\right.}=\frac{\mu_{\text {source }}}{26.9}$. 
Table 1. Unidentified 3EG sources without known possible galactic counterparts

\begin{tabular}{|c|c|c|c|c|c|c|c|c|c|}
\hline $\begin{array}{l}\gamma \text {-Source } \\
(3 \mathrm{EG} \mathrm{J})\end{array}$ & $\begin{array}{c}l \\
(\mathrm{deg})\end{array}$ & $\begin{array}{r}b \\
(\operatorname{deg})\end{array}$ & $\Delta \theta$ & $\Gamma$ & $\begin{array}{c}\left\langle F_{\gamma}\right\rangle 1^{-8} \\
\left(\mathrm{ph} \mathrm{cm}^{-2} \mathrm{~s}^{-1}\right)\end{array}$ & $\sigma_{\mathrm{sd}}$ & $N_{\mathrm{vp}}$ & $\mu$ & $I$ \\
\hline $0241+6103^{\star}$ & 135.87 & 0.99 & 0.18 & $2.21 \pm 0.07$ & 69.4 & 24.6 & 9 & 35.4 & 1.31 \\
\hline $0323+5122$ & 145.64 & -4.67 & 0.55 & $2.38 \pm 0.41$ & 17.5 & 9.5 & 6 & 54.2 & 2.01 \\
\hline $0416+3650^{\dagger}$ & 162.22 & -9.97 & 0.63 & $2.59 \pm 0.32$ & 21.1 & 14.9 & 10 & 70.6 & 2.61 \\
\hline $0435+6137$ & 146.50 & 9.50 & 0.66 & $2.46 \pm 0.35$ & 18.8 & 5.1 & 4 & 27.1 & 1.00 \\
\hline $0459+3352$ & 170.30 & -5.38 & 0.98 & $2.54 \pm 0.24$ & 21.9 & 7.6 & 11 & 34.7 & 1.28 \\
\hline $0500+2529$ & 177.18 & -10.28 & 0.36 & $2.52 \pm 0.32$ & 14.2 & 8.4 & 17 & 59.1 & 2.19 \\
\hline $0510+5545$ & 153.99 & 9.42 & 0.71 & $2.19 \pm 0.20$ & 22.5 & 11.3 & 9 & 50.2 & 1.86 \\
\hline $0520+2556$ & 179.65 & -6.40 & 0.86 & $2.83 \pm 0.24$ & 25.0 & 8.5 & 13 & 30.0 & 1.11 \\
\hline $0521+2147$ & 183.08 & -8.43 & 0.45 & $2.48 \pm 0.15$ & 28.5 & 13.9 & 15 & 48.8 & 1.81 \\
\hline $0533+4751$ & 162.61 & 7.95 & 0.60 & $2.55 \pm 0.23$ & 18.2 & 6.1 & 8 & 33.5 & 1.24 \\
\hline $0546+3948$ & 170.75 & 5.74 & 0.67 & $2.85 \pm 0.21$ & 17.5 & 8.1 & 13 & 46.3 & 1.71 \\
\hline $0556+0409$ & 202.81 & -10.29 & 0.47 & $2.45 \pm 0.16$ & 19.3 & 8.8 & 10 & 45.6 & 1.69 \\
\hline $0628+1847$ & 193.66 & 3.64 & 0.57 & $2.30 \pm 0.10$ & 32.9 & 12.0 & 10 & 36.5 & 1.35 \\
\hline $0903-3531$ & 259.40 & 7.40 & 0.58 & $2.66 \pm 0.24$ & 22.4 & 9.4 & 6 & 41.9 & 1.55 \\
\hline $1014-5705$ & 282.80 & -0.51 & 0.67 & $2.23 \pm 0.20$ & 47.5 & 18.9 & 10 & 39.8 & 1.47 \\
\hline $1316-5244$ & 306.85 & 9.93 & 0.50 & $2.54 \pm 0.18$ & 19.7 & 11.8 & 11 & 59.8 & 2.21 \\
\hline $1631-4033$ & 341.61 & 5.24 & 0.89 & $2.25 \pm 0.27$ & 32.3 & 11.0 & 11 & 34.0 & 1.26 \\
\hline $1633-3216$ & 348.10 & 10.48 & 0.87 & $2.58 \pm 0.24$ & 16.1 & 9.5 & 12 & 59.0 & 2.18 \\
\hline $1638-5155$ & 334.05 & -3.34 & 0.68 & $2.56 \pm 0.21$ & 45.3 & 29.9 & 15 & 66.0 & 2.44 \\
\hline $1704-4732$ & 340.10 & -3.79 & 0.66 & $1.86 \pm 0.33$ & 29.5 & 23.6 & 18 & 80.0 & 2.96 \\
\hline $1717-2737$ & 357.67 & 5.95 & 0.64 & $2.23 \pm 0.15$ & 32.9 & 17.8 & 18 & 54.1 & 2.00 \\
\hline $1735-1500$ & 10.73 & 9.22 & 0.77 & $3.24 \pm 0.47$ & 19.0 & 45.4 & 19 & 238.9 & 8.86 \\
\hline $1736-2908$ & 358.79 & 1.56 & 0.62 & $2.18 \pm 0.12$ & 49.6 & 32.4 & 21 & 65.3 & 2.42 \\
\hline $1741-2050$ & 6.44 & 5.00 & 0.63 & $2.25 \pm 0.12$ & 33.4 & 19.1 & 19 & 57.2 & 2.12 \\
\hline $1741-2312$ & 4.42 & 3.76 & 0.57 & $2.49 \pm 0.14$ & 34.0 & 19.8 & 19 & 58.2 & 2.15 \\
\hline $1744-3934$ & 350.81 & -5.38 & 0.66 & $2.42 \pm 0.17$ & 26.5 & 21.8 & 20 & 82.2 & 3.04 \\
\hline $1746-1001$ & 16.34 & 9.64 & 0.76 & $2.55 \pm 0.18$ & 29.7 & 25.6 & 18 & 86.2 & 3.19 \\
\hline $1757-0711$ & 20.30 & 8.47 & 0.68 & $2.51 \pm 0.20$ & 33.6 & 18.2 & 15 & 54.2 & 2.01 \\
\hline $1800-0146$ & 25.49 & 10.39 & 0.77 & $2.79 \pm 0.22$ & 25.5 & 13.3 & 14 & 52.1 & 1.93 \\
\hline $1810-1032$ & 18.81 & 4.23 & 0.39 & $2.29 \pm 0.16$ & 31.5 & 22.2 & 17 & 70.5 & 2.61 \\
\hline $1812-1316$ & 16.70 & 2.39 & 0.39 & $2.29 \pm 0.11$ & 43.0 & 30.2 & 18 & 70.3 & 2.60 \\
\hline $1828+0142$ & 31.90 & 5.78 & 0.55 & $2.76 \pm 0.39$ & 30.8 & 44.3 & 8 & 143.8 & 5.33 \\
\hline $1834-2803$ & 5.92 & -8.97 & 0.52 & $2.62 \pm 0.20$ & 17.9 & 13.7 & 20 & 76.5 & 2.83 \\
\hline $1837-0606$ & 25.86 & 0.40 & 0.19 & $1.82 \pm 0.14$ & 57.5 & 37.4 & 12 & 65.0 & 2.41 \\
\hline $1904-1124$ & 24.22 & -8.12 & 0.50 & $2.60 \pm 0.21$ & 22.5 & 17.7 & 14 & 78.6 & 2.91 \\
\hline $1928+1733$ & 52.91 & 0.07 & 0.75 & $2.23 \pm 0.32$ & 38.6 & 41.6 & 10 & 107.7 & 3.99 \\
\hline $1958+2909$ & 66.23 & -0.16 & 0.57 & $1.85 \pm 0.20$ & 35.6 & 16.2 & 10 & 45.5 & 1.68 \\
\hline $2035+4441$ & 83.17 & 2.50 & 0.54 & $2.08 \pm 0.26$ & 39.1 & 35.4 & 14 & 90.5 & 3.35 \\
\hline $2100+6012^{\dagger}$ & 97.76 & 9.16 & 0.48 & $2.21 \pm 0.25$ & 23.3 & 8.7 & 7 & 37.3 & 1.38 \\
\hline $2206+6602^{\dagger}$ & 107.23 & 8.34 & 0.88 & $2.29 \pm 0.26$ & 25.4 & 4.6 & 4 & 18.1 & 0.67 \\
\hline
\end{tabular}

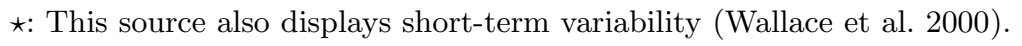

Table 2 shows the variability results for the pulsars in the 3EG catalog, whereas the last column of Table 1 shows the results of the variability index $I$ for our sample of unidentified 3EG sources.

The adopted variability criterion is then to consider that any source for which the $\mu$-value is less than the upper value of $\mu$ for pulsars $\left(\mu_{\max }=40.5\right)$, is a nonvariable source. At least, we can assure that for this kind of source, and with the data now at hand, it is not possible to discriminate any significant long-term variation in its gamma emission. We shall also consider that sources with $\mu$-values between 40.5 and 65 are dubious cases, and that sources with $\mu>65$ are variable. In terms of the averaged index $I$, this is equivalent to saying that variable sources will be those with $I>2.5$, which is $3 \sigma$ away from the statistical variability of pulsars. The value of $1 \sigma$ is naively obtained from the standard deviation of the $I$-index for pulsars. Since this is a population of just a few members, this forces us to be careful in assigning to a source the "variable" status.

We adopted this criterion, previously used in blazar variability analysis by some of us (Romero et al. 1994) and applied to 3EG sources by Zhang et al. (2000), rather than the similar one used by McLaughlin et al. (1996) and Wallace et al. (2000) in order to allow a direct comparison with the spurious statistical variability shown by 
Table 2. Statistical variability of pulsars. The last one was identified by Cusumano et al. (2000)

\begin{tabular}{llcrccccc}
\hline $\begin{array}{l}\gamma \text {-Source } \\
(3 \mathrm{EG} \mathrm{J})\end{array}$ & Pulsar Name & $\begin{array}{c}l \\
(\mathrm{deg})\end{array}$ & $\begin{array}{r}b \\
(\mathrm{deg})\end{array}$ & & $\begin{array}{c}\langle\Gamma\rangle \\
\left(\mathrm{ph} \mathrm{cm}^{-2} \mathrm{~s}^{-1}\right)\end{array}$ & & $\sigma_{\mathrm{sd}}$ & $\mu$ \\
\hline $0534+2200$ & Crab & 184.5 & -5.8 & 2.19 & 219.0 & 29.2 & 16 & 13.3 \\
$0633+1751$ & Geminga & 195.0 & 4.3 & 1.66 & 350.9 & 50.1 & 14 & 14.3 \\
$0834-4511$ & Vela & 263.5 & -2.8 & 1.69 & 848.2 & 183.6 & 8 & 21.6 \\
$1058-5234$ & PSR B1055-52 & 286.1 & 6.5 & 1.94 & 36.1 & 14.6 & 15 & 40.4 \\
$1710-4439$ & PSR B1706-44 & 343.0 & -2.8 & 1.86 & 109.9 & 44.5 & 20 & 40.5 \\
$0634+0521$ & & 206.1 & -1.4 & 2.03 & 25.5 & 7.2 & 9 & 27.6 \\
\hline
\end{tabular}

pulsars. Since the $I$-index establishes how variable a source is with respect to the pulsar population, it can be considered as indicative of how reliable we can be about the possible physical variation in the gamma emission. The combination of the results of Zhang et al. (2000) with those presented in this paper yields the variability index for all unidentified low-latitude sources in the 3EG catalog, see Appendix C. Note, however, that Zhang et al.'s study differs from the present one in that they considered PSR B1951+32 for the determination of $\left\langle\mu>_{\text {pulsars }}\right.$. This pulsar is not in the $3 \mathrm{EG}$ catalog. The fluxes used by Zhang et al. were taken from data reduced using a different technique. The inclusion of this pulsar makes $<\mu>_{\text {pulsars }}=77.7 \pm 50.0$ instead of the much lower value we obtain. This high value of $\langle\mu\rangle_{\text {pulsars }}$ causes the $I$-index for AGNs to be compatible with a non-variable population, and it is also the reason why the majority of Zhang et al.'s sources are non-variable ones. In Appendix C, the values of $I$ for all sources at low latitudes $\left(|b| \leq 10^{\circ}\right)$ are computed under our normalization.

A more comprehensive study on the long term variability (more than one month) of the gamma-ray sources was recently presented by Tompkins (1999). This work re-analyzed the EGRET data to take into account not only all sources included in the 3EG catalog, but also the 145 marginal sources that were detected but not included in the final official list. The maximum likelihood set of source fluxes was then computed. From those fluxes, a new statistics measuring the variability is defined as $\tau=\sigma / \mu$, where $\sigma$ is the standard deviation of the fluxes and $\mu$ their average value. The strength of this approach lies in that it takes into account possible fluctuations from the background and from neighboring sources, careful sensitivity corrections throughout EGRET lifetime, and others systematic errors. On the other hand, our method can be very useful as a first approach: when one takes a fairly safe assumption as the threshold for variability (such as the one introduced above, i.e. a source is classified as variable if $I>2.5$ ) it yields results that are compatible with Tompkins'. For instance, if we consider the sources that Tompkins found as the most variable ones, we find that only two of them are included in our sample. These have $I=2.96$ and $I=5.33$, respectively. If we now consider the sources that Tompkins classified as less variable, we find that two of them are in our sample, with indices $I=1.00$ and $I=1.86$. The rest of the cases are compatible too, but less strength should be put on the results, since most of them are dubious cases in both schemes. We then found that our method, avoiding most of the criticism of previous studies (Tompkins 1999) is well-fitted as a discriminator between variable and non-variable sources using the publicly available EGRET data.

With our adopted criterion, we find that only a low $25.0 \%$ of the sources in our sample are non-variable, $42.5 \%$ are dubious cases, and $32.5 \%$ are variable. In this latter group, three sources have a variability index $5 \sigma$ away from the statistical variations of pulsars. In Fig. 2 we present the histogram for the variability index $I$ in our sample, along with a Gaussian fit which presents its peak at $I=$ 2.0 and a standard deviation equal to $\sigma_{I}=0.7$. In order to better evaluate these results, we have also computed the variability index for the 66 confirmed AGNs in the 3EG catalog. A table with these results (with the same columns as in Table 1) is given in Appendix B. Fifty-eight AGNs appear to be variable sources. We show the distribution of the variability index for AGNs in Fig. 3. The Gaussian fit in this case peaks at $I=2.3$ and the standard deviation is $\sigma_{I}=0.8$. Since the distribution of the unidentified sources under study is similar to that obtained for AGNs, which is a well-known variable population, we can conclude that many of our EGRET sources may actually vary on timescales of years.

In Fig. 4 we plot the computed value of $I$ versus the observed value of the spectral index $\Gamma$, for the sources in our sample. We also show in the same figure the $I-\Gamma$ relation for AGNs. Two solid horizontal lines represent the lower and upper bound for the statistical variability of the pulsar population. An horizontal dashed line stands for the value of the variability indices above which the variability of the sources can be established (i.e. above $3 \sigma$ level). From these plots, it appears that more than a single population is present. Pulsars should be located at the lower left corner of the figure: they have a hard spectral index and are non-variable. A different group of sources, with spectral index in the range $\sim 2.1-\sim 2.6$ and being marginally variable or variable, appears towards the center of the figure. Most of the AGNs are located in this region of the plot. Finally, some sources seem to show a steepening in their spectral index with increasing values of variability. A Spearman correlation test for the variable sources yields a probability of about $8 \%$ for this being a pure chance effect. These sources could then be members 


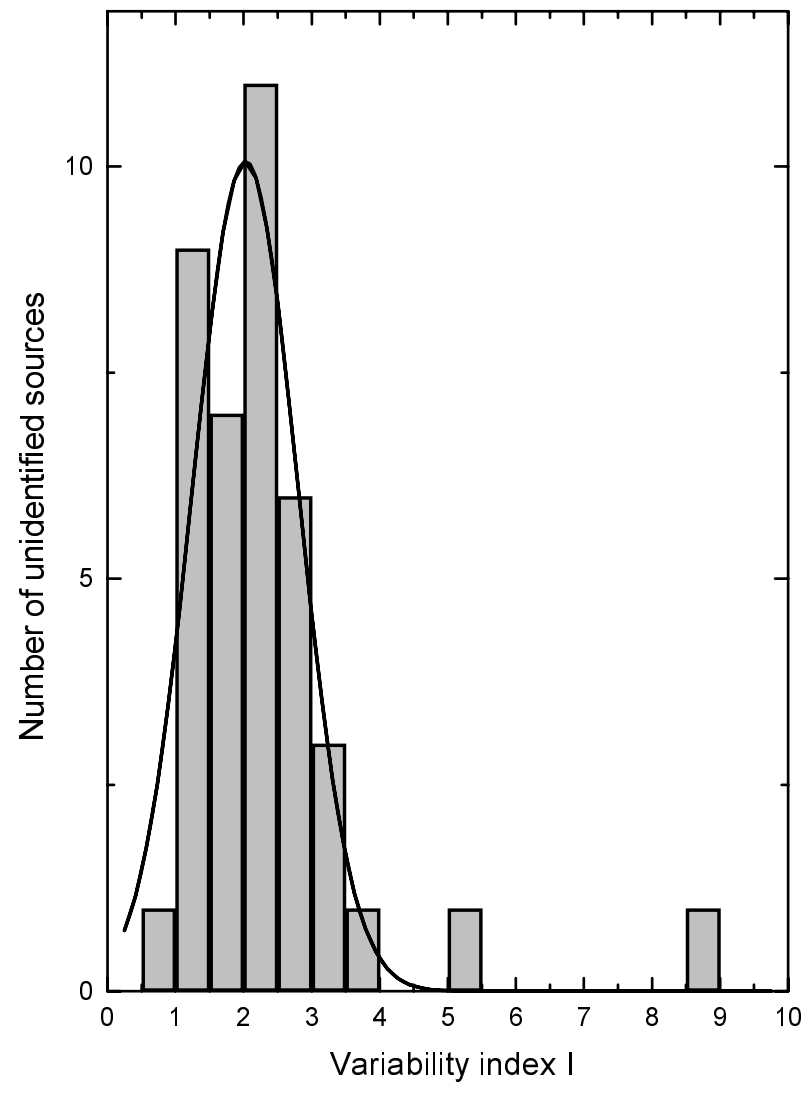

Fig. 2. Variability index distribution. The spline curve is the best Gaussian fit, it peaks at $I=2.0$ and presents a standard deviation of $\sigma_{I}=0.7$. These values suggest that many of the unidentified sources are variable

of a new population of galactic objects or, perhaps, they might be AGNs seen through the disk of the Galaxy.

Since AGNs are isotropically distributed, we can determine a mean value for the number of AGNs detected by EGRET per square degree: $N_{\mathrm{AGN}}=8.910^{-4}$ $\mathrm{AGN} /$ degree $^{2}$. At latitudes in the range $-10^{\circ} \leq b \leq 10^{\circ}$, the number of unidentified sources that are marginally variable or variable yields a number density $N_{\text {unid }}=$ $4.310^{-3}$ objects/degree ${ }^{2}$. This shows that there are five times more variable objects within the galactic plane than away from it. Then, we have to conclude that if the distribution of AGNs is isotropic, we have an excess of variable galactic sources representing a new class of population, as it was already noticed in previous studies by McLaughlin et al. (1996), by Mukherjee et al. (1997), by Tavani et al. (1997), and very recently by Wallace et al. (2000) ${ }^{1}$.

Considering the sample of the 2EG catalog, Özel \& Thompson (1996) argued that one half or more of the unidentified EGRET sources lying at high latitudes are of galactic origin. Nice \& Sawyer (1997) found no evidence that pulsars were the constituents of this putative galactic population. This agrees with our results, since we show here that this new class of galactic objects should

\footnotetext{
1 The latter work focuses only on very short timescales.
}

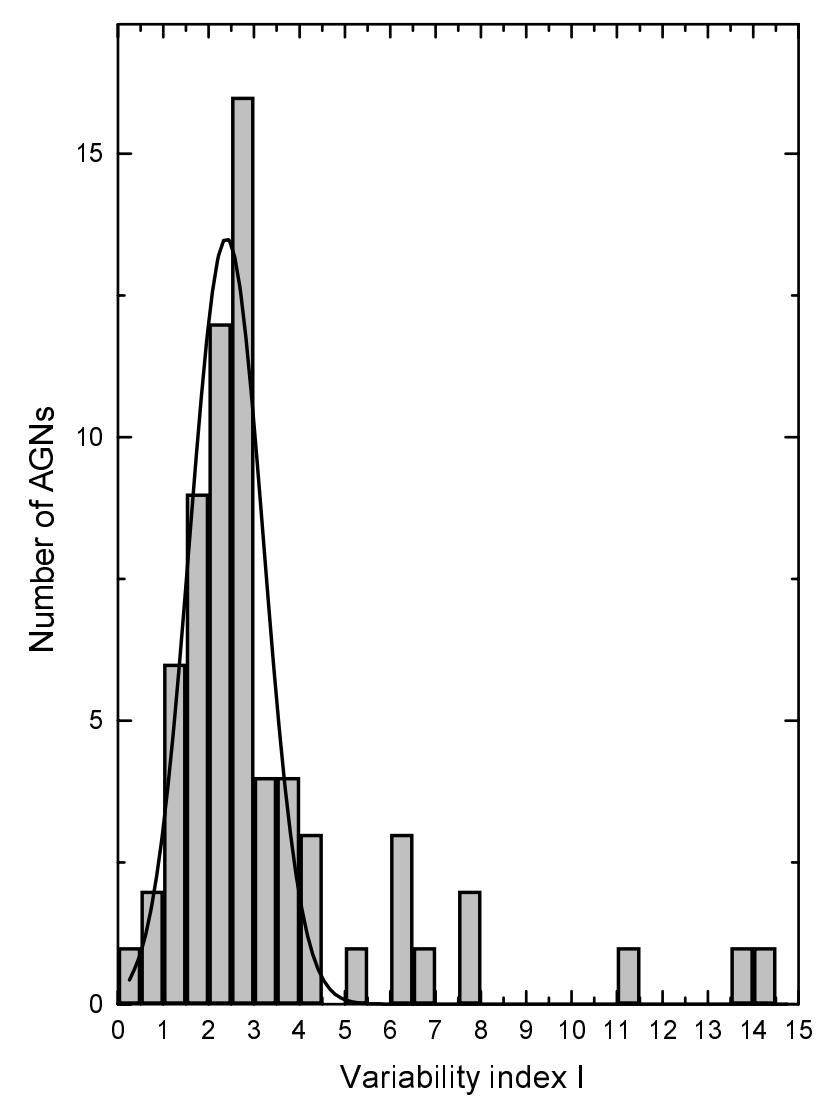

Fig. 3. Variability index distribution for the 66 AGNs in the 3EG catalog. The spline curve is the best Gaussian fit, it peaks at $I=2.3$ and has a standard deviation of $\sigma_{I}=0.8$

resemble, from the point of view of gamma-ray observations, the AGN population.

It is perhaps appropriate to come back to the discussion of what is a real source (as opposed to possible noise variations) in this study. We are considering that the $3 \mathrm{EG}$ catalog is composed of real sources, disregarding the level of flux detected in each case. The exception to this rule is the six artifacts related to the Vela pulsar, which disappear in a map where the Vela emission is suppressed (Hartman et al. 1999). However, since many detections in the EGRET catalog are below the $3 \sigma$ significance, and the fluxes are compatible with zero, a more careful treatment is in order. We can consider only those sources with $\sqrt{T S}>5 \sigma$, which implies a flux above $3010^{-8} \mathrm{ph} \mathrm{cm}^{-2} \mathrm{~s}^{-1}$. In that situation we are left only with 16 sources, four of them having $I>2.5$. One of these sources is $3 \mathrm{EG} 1828+0142$, with $I=5.33$, to which we devoted a separate work elsewhere (Punsly et al. 2000). If the flux threshold is lowered to $1810^{-8} \mathrm{ph} \mathrm{cm}^{-2} \mathrm{~s}^{-1}$, we are left with 35 sources, and now 11 of them have $I>2.5$. Within this group is 3EG 1735-1500, which has $I=8.86$. It is then apparent that variable sources exist even when the flux threshold is raised.

The relevance of the previous point lies mainly in the galactic longitude distribution of the 40 3EG sources of Table 1. In the left panel of Fig. 5 we show the histogram distribution with galactic longitude of all sources in our 


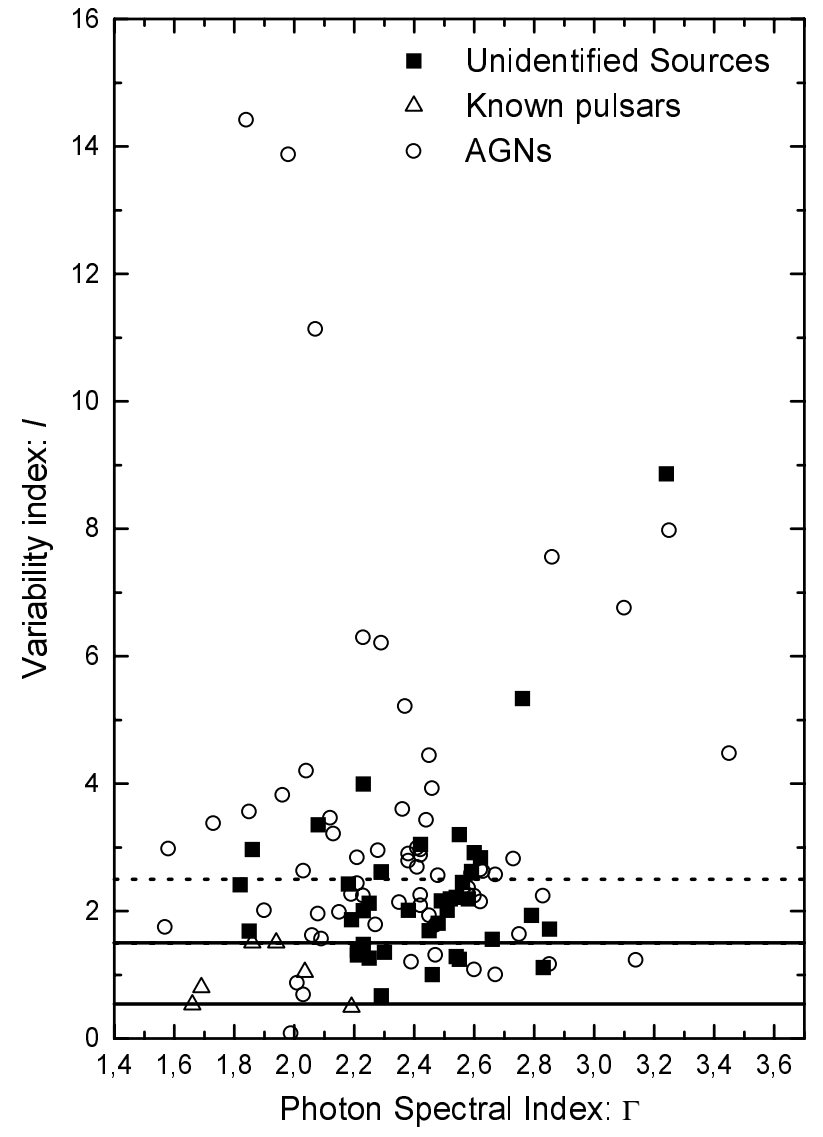

Fig. 4. Variability versus photon spectral index plot. We also present the values for pulsars and 66 AGNs in the 3EG catalog. Horizontal solid lines represent the lowest and maximum values for pulsars. The dotted line corresponds to the limit above which all sources are variable $(3 \sigma)$

sample; it is clear that it is peaked towards the galactic center and the galactic anti-center. Although it is true that the EGRET exposure was greater in these regions, it appears that the number of sources of this sample that were detected at these positions is relatively larger than what results when the whole $3 \mathrm{EG}$ catalog is considered. If we take a more strict limit on the flux, such as given by taking sources only with $\sqrt{T S}>5 \sigma$, we obtain the distribution shown on the right panel. That is, a more restrictive limit on the adopted minimum flux will preferentially delete the anti-center sources, leaving strong detections towards the galactic center, which is certainly more compatible with a real galactic population.

It seems to be possible that at least a part of this excess in the number of sources towards the galactic center could be the low-latitude tail of the new population found by Gehrels et al. (2000) at mid-latitudes. This speculation is supported by the fact that the average spectral index of the sources associated with the Gould belt is $2.49 \pm 0.04$, quite compatible with the indices of the sources in the sample here considered. However, the sources in the midlatitude population are weaker than many of the variable and strong ones of our group. If stars are responsible for the weaker sources, as suggested by Gehrels et al. (2000)

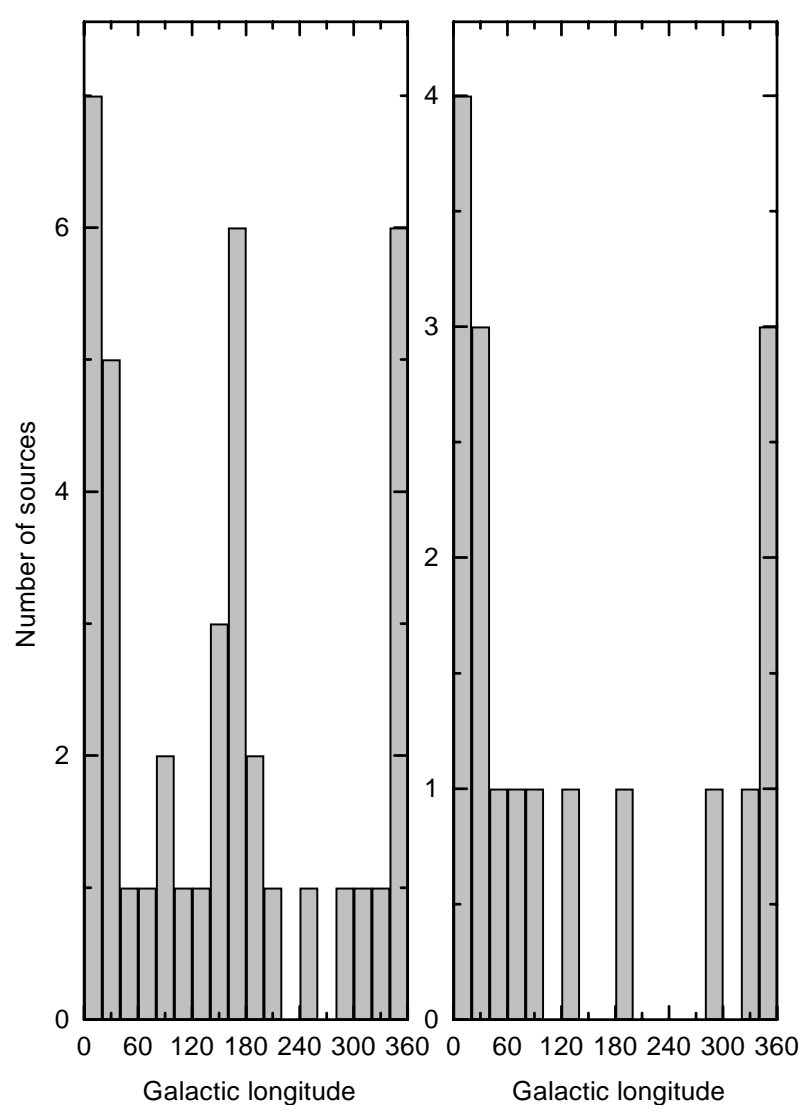

Fig. 5. Galactic longitude distribution of the 3EG sources considered in our sample, for different flux lower limits. See text for explanation

and by Benaglia et al. (2000), then a different type of object, intrinsically much more luminous at gammarays, should be behind the stronger low-latitudes EGRET detections.

A preliminary conclusion of this section could then be stated as follows: a new type of galactic objects may be needed to explain the behaviour of some unidentified 3EG sources at low latitudes. This population should be able to display high luminosities, steep gamma-ray spectral indices and significant gamma-ray variability. Figure 4 suggests that our sample, although mostly of galactic origin, mimics some observational properties of AGNs.

\section{Uncatalogued supernova remnants}

We consider in this section whether some of the sources in our sample may be associated with recently proposed candidates to supernova remnants, not catalogued by Green (1998). Our interest in this search resides in the fact that young stellar objects like recently formed black holes and pulsars can still be associated with the gaseous remnant of the original supernova that created them.

The diffuse non-thermal emission of the galactic disk, formed by the interaction of the leptonic component of the cosmic rays with the galactic magnetic field, is surely veiling many remnants of low surface brightness. Recent observational studies using filtering techniques in the 
analysis of radio data have revealed several new SNR candidates that are not yet included in the latest issue of Green's catalog, which was used in the study presented in Paper I (e.g. Duncan et al. 1995; Combi \& Romero 1998; Combi et al. 1998b, 1999a; Jonas 1999). In general, these new candidates are much more extended than those previously known. There are 101 of these weak non-thermal structures detected so far in the Galaxy. This number significantly extends Green's (1998) catalogue. The list of these new candidates, compiled from the papers and studies above mentioned is being published electronically as an Appendix.

With the aim of finding the positional coincidences between our sample of 40 3EG unidentified sources and the uncatalogued candidates to SNRs, we used the code developed in Paper I. We have found that only 7 gammaray sources in our sample are positionally coincident with non-thermal radio structures. The positional coincidences thus obtained are shown in Table 3, where we give the name of the gamma-ray sources, the central position of the SNR candidates, the distance from the EGRET source to the centre of the radio structure $(\delta)$, and the addition of the radius of the extended sources and the uncertainty in the position of the EGRET detections $(\Delta)$.

To estimate the statistical significance of these coincidences, we have numerically simulated a large number of synthetic sets of EGRET sources using the code described in Paper I. As in that paper, the simulations were constrained to preserve the original gradient in the number distribution of unidentified gamma-ray sources towards the galactic plane. The results of this study show that the expected number of chance associations is $10.4 \pm 2.7$, quite compatible with, and even bigger than, the actual result. We conclude, then, that there is no statistical evidence suggesting that the 3EG sources analyzed in our sample are associated with known or potential SNRs.

\section{Search for extended radio counterparts in selected sources}

We have especially examined the radio sky towards the best estimate positions of the unidentified 3EG sources J0241+6103, J0435+6137, J0628+1847, J10145705, J1631-4033, J1735-1500, J1828+0142, J1928+1733, J2035+4441 and J2206+66. From Table 1, it can be seen that these sources can be grouped into two sets, at the extremes of the variability and spectral index ranges. On one hand, we have six sources with low variability and relatively hard spectral indices. These sources could, in principle, be associated with pulsars or with unknown new SNR candidates. Only one of them, 3EG J1631-4033, presented positional superposition with a known candidate to SNR (see Table 3). There are, on the other hand, four sources with high variability ( $I$-values ranging from 3.3 to 8.8) and steep spectral index ( $\Gamma$ ranging from 2.08 to $3.24)$.
Table 3. Positional coincidences with candidates to SNRs

\begin{tabular}{lcrcc}
\hline $\begin{array}{l}\gamma \text {-Source } \\
(3 \mathrm{EG} \mathrm{J})\end{array}$ & $\begin{array}{c}l \\
(\mathrm{deg})\end{array}$ & $\begin{array}{c}b \\
(\mathrm{deg})\end{array}$ & $\begin{array}{c}\delta \\
(\mathrm{deg})\end{array}$ & $\begin{array}{c}\Delta \\
(\mathrm{deg})\end{array}$ \\
\hline $0903-3531$ & 260.20 & 1.40 & 6.05 & 13.58 \\
$1631-4033$ & 342.60 & 8.20 & 3.11 & 3.89 \\
$1638-5155$ & 333.00 & 0.00 & 3.50 & 3.68 \\
$1704-4732$ & 340.80 & -4.80 & 1.22 & 2.11 \\
$1717-2737$ & 356.90 & 8.50 & 2.66 & 5.39 \\
$1834-2803$ & 7.30 & -5.30 & 3.91 & 4.12 \\
$1837-0606$ & 27.00 & 0.50 & 1.14 & 3.69 \\
\hline
\end{tabular}

We used continuum radio data at 1420 and $2400 \mathrm{MHz}$, from the surveys by Reich \& Reich (1986), and Duncan et al. (1995), in order to study the possible presence of weak and extended radio sources within the EGRET positional error boxes of this set. Specifically, we searched for yet undetected low-brightness SNRs that could be associated with the pulsars or the black holes created in the original supernova explosion. We remove the background diffuse radiation using the Gaussian filtering technique described in a recent series of papers by Combi and coworkers (e.g. Combi \& Romero 1995, 1998; Combi et al. 1998a,b, 1999a,b). The reader is referred to these papers for details. Three of the fields that were studied presented features interesting enough to deserve individual description. Below we briefly comment on two of them: 3EG J0435+6137 and 3EG J1631-40. The source 3EG J1828+01 is discussed separately in the paper by Punsly et al. (2000). The remaining fields were empty of extended radio structures or too confused even after background subtraction as to provide reliable information.

\section{1. $3 E G ~ J 0435+6137$}

In Fig. 5, upper panel, we show the filtered radio image of the region around 3EG J0435+6137 at $1.42 \mathrm{GHz}$ (with an rms noise of $20 \mathrm{mK}$ and angular resolution of $\left.34^{\prime}\right)$ super-imposed to the EGRET probability contours. Two apparently extended radio sources are visible in the field. The strongest one, $87 \mathrm{~GB} \mathrm{~J} 0443+6118$, is partially outside the $95 \%$ confidence contour of the gamma-ray detection. Another source, named 87GB J0441+6145, is located $20^{\prime}$ away from the best estimated position of 3 EG J0435+6137, well within the $95 \%$ confidence contour.

In Fig. 6, lower panel, we show the central region of the radio field at $5 \mathrm{GHz}$ and a resolution of $3.5^{\prime}$ (Condon et al. 1989). Several point-sources are visible in the frame. The identification of the main ones is also indicated in the figure. None of these sources is known to be extragalactic. One of them, 87GB J0442+6140, is coincident with the X-ray source 1RXS J044239.3+61404. Another source, 87GB J0435+6137 (also known as TXS $0431+615)$, is located exactly at the best estimated position of $3 \mathrm{EG}$ J0435+6137. This source has a steep spectral index of $\sim-1.2$. 


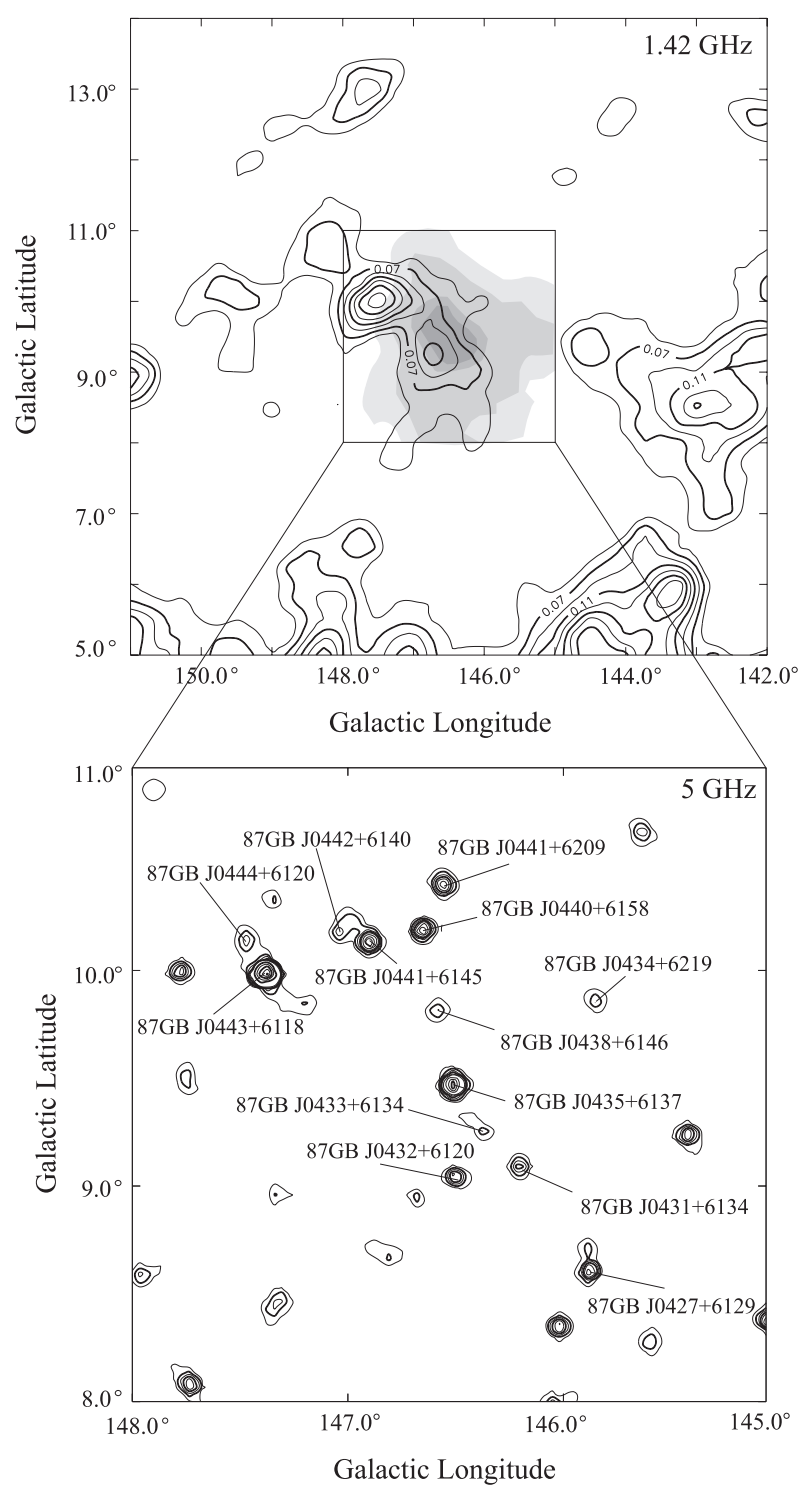

Fig. 6. Upper panel: map of the radio field around 3EG J0435+6137 at $1.42 \mathrm{GHz}$ (angular resolution of $\left.34^{\prime}\right)$ with the diffuse emission filtered using a beamwidth of $120^{\prime} \times$ $120^{\prime}$. Contours are labeled in steps of $0.02 \mathrm{~K}$ from 0.05 to $0.22 \mathrm{~K}$ in brightness temperature. The superimposed grey-scaled levels represent the $99 \%, 95 \%, 68 \%$, and $50 \%$ statistical probability that the gamma-ray source lies within each contour. Lower panel: image at $5 \mathrm{GHz}$ of the central field obtained with the former NRAO 91-m telescope at Green Bank (Condon et al. 1994). Contours are shown starting at $10 \mathrm{mJy}^{\text {beam }}{ }^{-1}$, in steps of $10 \mathrm{mJy}^{\text {beam }^{-1}}$

We have made Gaussian convolutions of those groups of point sources that are nearly coincident with the apparent extended sources seen in the lower resolution map of the upper panel, using a beamwidth of $34^{\prime}$. We have found that both apparent structures are likely artifacts resulting from the merging of the several point-like sources in the region and that, consequently, no extended nonthermal source can be associated with 3EG J0435+6137. The counterpart, if it exists at all, should be one of the point sources. The most promising candidates seems to be $87 \mathrm{~GB}$ J0442+6140, which is also detected at X-rays, and $87 \mathrm{~GB}$ J0435+6137, which is the strongest one at $5 \mathrm{GHz}$.

\section{2. $3 E G ~ J 1631-4033$}

Figure 7 shows the filtered radio continuum map of the field around 3EG J1631-4033 at $2.4 \mathrm{GHz}$ (with an rms noise of $12 \mathrm{mJy}$ beam $^{-1}$ and an angular resolution of $\left.10.4^{\prime}\right)$. The confidence contours of the likelihood test statistics of the EGRET detection are also shown as a superposed gray-scale. Four point-like radio sources can be identified with PMN sources (Griffith \& Wright 1993), namely (listed by increasing Galactic longitude), PMN J1627-3952 (noted as S1 in Fig. 7), PMN J1631-4015 (S2), PMN J1636-4101 (S3), and PMN J1631-3956 (S4). The angular separation between the location of the highest likelihood test statistic for the gamma-ray source and the position of PMN J1631-4015 is just $\sim 22$ arcmin. The strongest source, located at $(l, b) \sim\left(341.9^{\circ},+4.15^{\circ}\right)$, can be identified with PMN J1636-4101. We summarize the radio information on the point sources in Table 4 . Any of these sources can be considered as a potential counterpart. Additional and weaker sources in this region are listed in Table 5 (see below).

Another interesting feature revealed by the radio map is the existence of a minimum in the continuum emission towards the centre of the field at $(l, b) \sim\left(341.2^{\circ},+4.6^{\circ}\right)$. This might be the relic of an old explosive event in the ISM in this region. 
Table 4. Characteristics of the point-like radio sources inside the $\gamma$-ray contour of 3EG J1631-4033

\begin{tabular}{cccccccc}
\hline Source & $\begin{array}{c}(l, b) \\
(\mathrm{deg}, \mathrm{deg})\end{array}$ & $\begin{array}{c}F_{2.4 \mathrm{GHz}} \\
(\mathrm{mJy})\end{array}$ & $\begin{array}{c}F_{4.8 \mathrm{GHz}} \\
(\mathrm{mJy})\end{array}$ & $\begin{array}{c}F_{8.3 \mathrm{GHz}} \\
(\mathrm{mJy})\end{array}$ & $\alpha_{2.4 / 4.8}$ & $\alpha_{4.8 / 8.3}$ & ID \\
\hline $\mathrm{S} 1$ & $(341.6,+6.2)$ & 877.3 & 670.1 & - & -0.3 & - & PMN J1631-4015 \\
$\mathrm{S} 2$ & $(341.8,+5.4)$ & 892.1 & 490.0 & 150.2 & -0.8 & -2.0 & PMN J1627-3952 \\
$\mathrm{S} 3$ & $(341.9,+4.1)$ & 2260.3 & 1120.0 & 700.0 & -1.0 & -0.8 & PMN J1636-4101 \\
$\mathrm{S} 4$ & $(342.1,+5.6)$ & 520.2 & 180.0 & 50.0 & -1.5 & -2.2 & PMN J1631-3956 \\
\hline
\end{tabular}

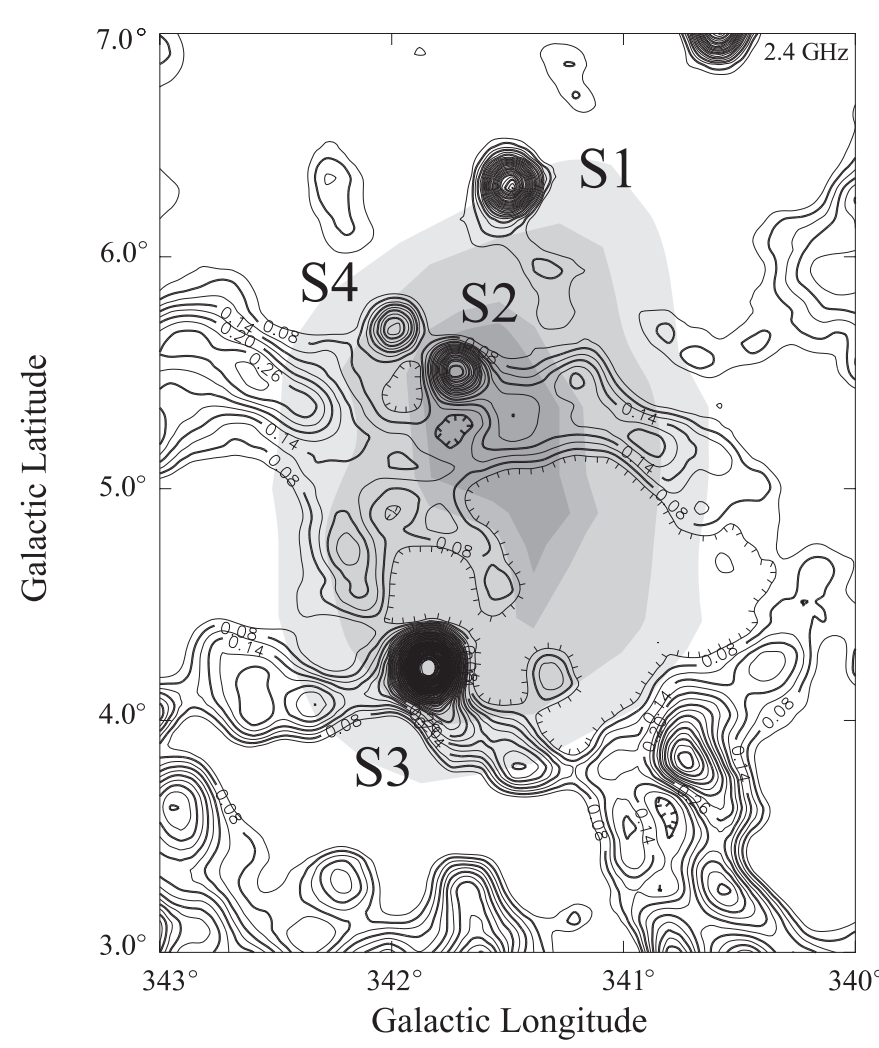

Fig. 7. Radio image of the field of 3EG J1631-40 at $2.4 \mathrm{GHz}$ (angular resolution of $10.4^{\prime}$ ) after the subtraction of the diffuse emission. Superimposed on the radio image we show the confidence contours of the likelihood test statistics of the EGRET detection. The best result for revealing a counterpart of the gamma-ray source is obtained for a filtering beamwidth of $90^{\prime} \times 90^{\prime}$ and 6 iterations. Radio contours are shown at steps of 0.05 from 0.05 to $6 \mathrm{Jy}$ beam $^{-1}$

\section{Point-like counterparts: Systematic search}

We have searched CATS (Verkhodanov et al. 1997), SIMBAD and NED databases for any possible radio counterparts for the gamma-ray sources in our sample. We have prepared a table containing the results of this search. This table (Table 5, which is published only in electronic form due to its length) lists all significant point-like radio sources, with flux densities above $30 \mathrm{mJy}$ at least at one wavelength, found within the $95 \%$ EGRET confidence contours of the 40 gamma-ray sources of Table 1.

In Table 5 there are entries for each gamma-ray source listing, from left to right, radio survey used, source name, right ascension and declination with the corresponding uncertainties, observing frequency, flux density and its error, and comments. The different fields are separated, and within each field (listed in increasing observing frequency), a different paragraph corresponds to each radio source. All listed sources (about 600, ranging from just a few to almost 50 per $3 \mathrm{EG}$ field) are located within the EGRET $95 \%$ confidence contours. The material contained in this table could be very useful for future research in this field.

\section{Discussion: The black hole hypothesis}

The existence of a number of variable gamma-ray sources with steep spectra and without strong radio or X-ray counterparts can be explained postulating a galactic population of magnetized black holes (Punsly 1998a,b, 1999). In these objects, strong magnetic fields $\left(\sim 10^{11} \mathrm{G}\right)$ are supported by an orbiting charged ring or disk. The black hole itself is also charged and rotating (Kerr-Newman black hole), but the entire configuration has zero net charge, in such a way that it is not quickly neutralized by the accretion of diffuse interstellar matter. The resulting object has a magnetosphere similar to that presented by pulsars. However, because of the absence of a solid surface, thermal X-rays are not produced, as in a neutron star. In addition, there is no accretion disk capable of generating strong X-ray emission as is expected in microquasars (Mirabel \& Rodriguez 1998). The radiation of the system, which is non-pulsating due to the alignment of the rotation and magnetic axes, is produced by synchroton and inverse Compton mechanism in two relativistic electronpositron jets which propagate along the rotation axis in opposite directions, as it occurs in AGNs. Contrary to the case of most AGNs, no redshifted emission lines should be present and, in contrast to BL Lacs, no weak nebulosity is expected in the optical band. Punsly et al. (2000) have recently shown that, when pair annihilation effects are taken into account in the calculation of the spectral energy distribution presented by magnetized black holes, a steep gamma-ray spectrum is produced. Variability naturally results in the model as the effect of shocks and firehose instabilities which induce large changes in the Doppler enhancement factor of the relativistic jets (Punsly et al. 2000).

At high energies, self-Compton losses provide most of the gamma-ray luminosity, reaching values in the range $10^{34}-10^{35} \mathrm{erg} \mathrm{s}^{-1}$ for black holes of a few solar masses and polar magnetic fields of $\sim 10^{11} \mathrm{G}$ (see Punsly et al. 2000 for details). If such an object is relatively close (e.g. $\sim 1 \mathrm{kpc}$ ), it could appear as a typical unidentified EGRET source with $\Gamma \sim 2.5$ or steeper. Although the spectral energy distribution is broad, only weak emission at longer wavelengths is expected. Radio counterparts, for instance, 
would present flux densities on the order of a few to a few tens of mJy at $5 \mathrm{GHz}$. Interestingly, one would also expect a mild correlation in variable gamma-ray sources between the variability and spectral indices, as seemingly observed in Fig. 4. See the work by Punsly et al. (2000) for details on the model.

Apart from 3EG J1828+0142, the source $3 \mathrm{EG} \quad \mathrm{J} 0241+6103 \quad$ (2EG J0241+6119) was already suggested as a possible identification of a magnetized black hole by one of us (Punsly 1999a,b). The source $2 \mathrm{EG} \mathrm{J} 0241+6119$ is known to be variable since the work by Tavani et al. (1998), who discarded a pulsar origin. This source appeared in a search for gamma-ray emitters, with strong emission also in X-rays, but weak output in radio. The binary system LSI $+61^{\circ} 303$, which is within the $95 \%$ probability contour of the gamma-ray detection, contains an unseen compact object suggested as the possible generator of the observed gamma rays (Punsly 1999a,b). However, a positive identification with the 3EG detection is not conclusive because of the fact that there is no signature in the gamma emission that can be correlated with the binary dynamics. Note that the magnetized black hole model in Punsly (1999b) does not produce any modulation of the gamma-ray signal on orbital time scales to a first order.

\section{Conclusions}

In this paper we have shown that there exists a group of unidentified, low-latitude, gamma-ray sources with the following characteristics:

1. They do not positionally coincide with any known galactic object such as Wolf-Rayet or Of massive stars, catalogued SNRs, and OB associations. The probability that a set of 40 sources, with the same positional errors as the EGRET ones, lacks any positional correlation with these galactic objects totally by chance is less than $310^{-4}$;

2. Even considering positional coincidences with 101 identified candidates to SNRs, in addition to Green's (1998) catalog, only 7 out of 40 sources present correlation, which is consistent with being the mere effect of chance;

3. The sample presents interesting variability features: $32 \%$ are variable and $45 \%$ of them are dubious cases;

4. The sample presents a steep average spectral index $<\Gamma>=2.41 \pm 0.2$; only 3 out of 40 sources have $\Gamma<2$;

5. Due to isotropy constraints on the average density of active galactic nuclei, not all of the 40 sources considered here can be AGNs seen through the disk of galaxy. The surface density of variable unidentified sources at low galactic latitudes is five times higher than that of AGNs out of the plane. This, and the fact that there are 5 AGNs already detected at $|b| \leq 10^{\circ}$, makes that if the distribution of AGNs is isotropic, just a few out of 40 gamma-ray sources could be AGNs. Indeed, if we extrapolate the AGN number density out of the plane to latitudes within $\left(-10^{\circ}, 10^{\circ}\right)$, we would expect 6.5 AGNs to be detected by EGRET, leaving room for just a couple of remaining unidentified objects to be AGNs, one, perhaps, being B2013+370 (Mukherjee et al. 2000).

Altogether, these features are not in accordance with usual models of gamma-ray emitters: they cannot be explained by pulsars, SNRs in interaction with nearby clouds, massive stars, or AGNs. In addition, we have found an apparent trend for sources with a higher degree of variability to present steeper photon spectral indices. This latter result, however, needs confirmation with the more complete sample to be obtained by the next generation of gamma-ray satellites (Gehrels et al. 1999). With the currently large positional error boxes, we have no hope to completely identify the source for all these detections, but if, after the launch of future gamma-ray satellites the previously quoted characteristics are confirmed, a new population of objects probably will be needed.

To our knowledge, the only model capable of reproducing all the peculiar features presented by some of the sources in our sample (steep spectral gamma-ray index, high variability, absence of clear lower-frequency counterparts, etc.) is that of isolated Kerr-Newman black holes (Punsly et al. 2000). This does not exclude the possibility that alternative models based on, for instance, stellar high-energy variability (Benaglia et al. 2000), or pulsar abnormal activity (e.g. Tavani \& Arons 1997), could play an important role explaining some detections.

The hypothesis of a population of magnetized black holes in the Galaxy can be tested with the forthcoming GLAST and INTEGRAL satellites, which will allow investigators to increase the sample and measure the spectral energy distribution for particular sources with high precision. In the case of INTEGRAL, the spectrometer SPI and the imager IBIS could detect the electronpositron annihilation features of the black hole jets, as indicated by Punsly et al. (2000).

Acknowledgements. This work was partially supported by CONICET (D.F.T., G.E.R., J.A.C., and P.B.), ANPCT (PICT 98, No. 03-04881), and by Fundación Antorchas, through separate grants to D.F.T., G.E.R., and J.A.C. H.A. thanks CONACyT (Mexico) for financial support under grant 27602-E. This research made use of the NASA/IPAC Extragalactic Database (NED) which is operated by the Jet Propulsion Laboratory, California Institute of Technology, under contract with the National Aeronautics and Space Administration. Tables are also available on-line at http://www.iar.unlp.edu.ar/garra. We gratefully acknowledge criticism and comments from an anonymous referee, which resulted in a substantial improvement of the paper.

\section{Appendix A: SNR candidates}

In this Appendix we present the complete list of 101 candidates to SNRs that were used in our study of positional coincidences. This list is presented electronically in 
Table 6 and it consists of three columns: galactic coordinates $(l, b)$ and the diameter of the extended source $(\theta)$ in arcminutes. The positions of these sources were reported by Duncan et al. (1995), Combi \& Romero (1998), Combi et al. (1998b, 1999a), and Jonas (1999). In general, these new candidates are much more extended and weaker than those previously known.

\section{Appendix B: Variability of AGNs}

In this Appendix we present data for the variability indices of the 66 AGNs already detected in the 3EG catalog. These data were used both in Fig. 4 and for making a comparison with our sample in the main text. We provide electronically (Table 7 ) the same columns as those given in Table 1 for the unidentified sources.

\section{Appendix C: Variability analysis of all low latitude gamma-ray sources}

If we combine the present work with that of Zhang et al. (2000), (taking into account the same normalization for $<\mu>_{\text {pulsars }}$, as explained above) who studied the variability of the sources which were positionally coincident with OB associations and SNRs as discovered by Romero et al. (1999), we can now make an assessment of the variability of the whole sample of unidentified sources at low galactic latitudes. There are 81 such sources, six of them (3EG J0824-4610, 0827-4247, 0828-4954, 0841-4356, 0859-4257 and 0848-4429) were recently reported to be artifacts produced by the high intensity emission of the nearby Vela pulsar. Another one, 3EG J0747-3412, was found to be coincident only with a WR star by Romero et al. (1999), and its variability index is here reported for the first time to be $I=2.81$. For future reference we present electronically in Table 8 the $3 \mathrm{EG}$ source name and the variability index $I$ for all low-latitude unidentified sources, normalized to the gamma-ray pulsars detected in the $3 \mathrm{EG}$ catalog.

\section{References}

Benaglia, P., Romero, G. E., Stevens, I., \& Torres, D. F. 2001, A\&A, 366, 605

Cassé, M., \& Paul, J. A. 1980, ApJ, 237, 236

Combi, J. A., \& Romero, G. E. 1995, A\&A, 303, 873

Combi, J. A., \& Romero, G. E. 1998, A\&AS, 128, 423

Combi, J. A., Romero, G. E., \& Benaglia, P. 1998a, A\&A, 333, L91

Combi, J. A., Romero, G. E., \& Arnal, M. 1998b, A\&A, 333, 298

Combi, J. A., Romero, G. E., \& Benaglia, P. 1999a, AJ, 118, 659

Combi, J. A., Romero, G. E., \& Benaglia, P. 1999b, ApJ, 519, L177

Condon, J. J., Broderick, J. J., \& Seielstad, G. A. 1989, AJ, 97,1064
Condon, J. J., Broderick, J. J., Seielstad, G. A., Douglas, K., \& Gregory, P. C. 1994, AJ, 107, 1829

Cusumano, G., Maccarone, M. C., Nicastro, L., Sacco, B., \& Kaaret, P. 2000, ApJ, 528, L25

Duncan, A. R., et al. 1995, MNRAS, 277, 36

Esposito, J. A., Hunter, S. D., Kanbach, G., \& Sreekumar, P. 1996, ApJ, 461, 820

Gehrels, N., \& Michelson, B. 1999, Astroparticle Phys., 11, 277

Gehrels, N., Macomb, D. J., Bertsch, D. L., Thompson, D. J., \& Hartman, R. C. 2000, Nature, 404, 363

Griffith, M. R., \& Wright, A. E. 1993, AJ, 105, 1666

Green, D. A. 1998, A Catalogue of Galactic Supernova Remnants, Mullard Radio Astronomy Observatory, Cambridge, UK (available at http://www.mrao.cam.ac.uk/surveys/snrs/)

Grenier, I. A. 1995, Adv. Space. Res., 15, 73

Hartman, R. C., Bertsch, D. L., Bloom, S. D., et al. 1999, ApJS, 123, 79

Hartman, R. C. 2000, private communication

Jonas, J. 1999, Ph.D. Thesis, Rhodes University, South Africa

Kaspi, V. M., Lackey, J. R., Mattox, J., et al. 2000, ApJ, 528, 445

McLaughlin, M. A., Mattox, J. R., Cordes, J. M., \& Thompson, D. J. 1996, ApJ, 473, 763

Mirabel, I. F., \& Rodríguez, L. F. 1998, Nature, 392, 673

Montmerle, T. 1979, ApJ, 231, 95

Mukherjee, R., Grenier, I. A., \& Thompson, D. J. 1997, Proceedings of the Fourth Compton Symposium, ed. C. D. Dermer, M. S. Strickman, \& J. D. Kurfess (AIP, New York), 384

Mukherjee, R., Gotthelf, E. V., Halpern, J., \& Tavani, M. 2000, ApJ, to appear [astro-ph/0005491]

Nice, D., \& Sawyer, T. 1997, ApJ, 476, 261

Özel, M. E., \& Thompson, D. J., 1996, ApJ, 463, 105

Punsly, B. 1998a, ApJ, 498, 640

Punsly, B. 1998b, ApJ, 498, 660

Punsly, B. 1999a, ApJ, 516, 141

Punsly, B. 1999b, ApJ, 519, 336

Punsly, B., Romero, G. E., Torres, D. F., \& Combi, J. A. 2000, A\&A, 364, 552

Reich, W., \& Reich, P. 1986, A\&AS, 63, 205

Romero, G. E., Combi, J. A., \& Colomb, F. R. 1994, A\&A, 288,731

Romero, G. E., Benaglia, P., \& Torres, D. F. 1999, A\&A, 348, 868, Paper I

Sturner, S. J., \& Dermer, C. D. 1995, A\&A, 293, L17

Tavani, M., Mukherjee, R., Mattox, J. R., et al. 1997, ApJ, 479, L109

Tavani, M., \& Arons, J. 1997, ApJ, 477, 439

Tavani, M., Kniffen, D., Mattox, J. R., Paredes, J. M., \& Foster, R. 1998, ApJ, 497, L89

Thompson, D. J., Arzoumanian, Z., Bertsch, D. L., et al. 1994, ApJ, 436, 229

Thompson, D. J., Bertsch, D. L., Dingus, B. L., et al. 1995, ApJS, 101, 259

Thompson, D. J., Bertsch, D. L., Dingus, B. L., et al. 1996, ApJS, 107, 227

Tompkins, W. 1999, Ph.D. Thesis, Stanford University

Verkhodanov, O. V., Trushkin, S. A., Andernach, H., \& Chernenkov, V. N. 1997, in ASP Conf. Ser. 125, Astronomical Data Analysis Software and Systems - VI, ed. G. Hunt, \& H. E. Payne, 322

Wallace, P. M., Griffis, N. J., Bertsch, D. L., et al. 2000, ApJ, 540,184

Yadigaroglu, I.-A., \& Romani, R. W. 1997, ApJ, 476, 356

Zhang, L., Zhang, Y. J., \& Cheng, K. S. 2000, A\&A, 357, 957 